“ (C) 2017 IEEE. Personal use of this material is permitted. Permission from IEEE must be obtained for all other uses, in any current or future media, including

reprinting/republishing this material for advertising or promotional purposes, creating new collective works, for resale or redistribution to servers or lists, or reuse of any copyrighted component of this work in other works." 


\title{
Theoretical Lower Bound for Indoor Visible Light Positioning Using Received Signal Strength Measurements and an Aperture-Based Receiver
}

\author{
Heidi Steendam, Senior Member, IEEE, Thomas Q. Wang and Jean Armstrong, Fellow, IEEE
}

\begin{abstract}
Indoor visible light positioning (VLP) using signals transmitted by lighting LEDs is a topic attracting increasing interest within the research community. In recent years, VLP techniques using a range of receiver structures and positioning algorithms have been described. In this paper we analyze the performance of a VLP system which uses an aperture-based receiver and measurements of received signal strength. An aperture-based receiver has a number of receiving elements each consisting of a photodiode and an associated aperture. It has been shown that receivers of this form can be designed which are compact and provide both a wide overall field-of-view and good angular diversity. As a result they can efficiently extract positionrelated information from light transmitted by non-directional LEDs. In our approach, we correlate the signals at the outputs of the photodiodes with a set of reference signals. The resulting observations include information on the received signal strength as well as the angle-of-arrival, and are used to directly estimate the receiver's position. In order to assess the performance of positioning algorithms based on this approach, we derive the Cramer-Rao lower bound on the position estimate. We show that the Cramer-Rao bound depends on the selected reference signal, and that sub-centimetre to centimetre accuracy can be obtained, using only a limited number of non-directional LEDs.
\end{abstract}

Index Terms-VLP, positioning, Cramer-Rao bound

\section{INTRODUCTION}

During the last decade, visible light communication (VLC) has received considerable attention in the research community, because it offers a low-cost alternative to traditional RF communication systems. The success of VLC is a consequence of the widespread deployment of white LEDs, which due to their low cost and energy efficiency, are gradually replacing conventional fluorescent and incandescent lights. In contrast to conventional light sources, white LEDs can be modulated at frequencies up to several $\mathrm{MHz}$, making high-speed data rate communication feasible [1]. Furthermore, VLC is a viable

Heidi Steendam is with the Digital Communications (DIGCOM) research group of the Telecommunications and Information Processing (TELIN) Department of Ghent University, Sint-Pietersnieuwstraat 41, 9000 Gent, Belgium. (E-mail: Heidi.Steendam@telin.ugent.be). Thomas Q. Wang is currently with the University of Technology Sydney, Sydney, Australia, but at the time the research was performed, he was with Monash University, Melbourne, Australia. (E-mail: qian.wang@uts.edu.au). Jean Armstrong is with the Department of Electrical and Computer Systems Engineering, Monash University, Melbourne, Australia, (Email: Jean.Armstrong@monash.edu)

The first author gratefully acknowledges the financial support from the Flemish Fund for Scientific Research (FWO). This research has been funded by the Interuniversity Attraction Poles Programme initiated by the Belgian Science Policy Office. This research was carried out during the sabbatical leave of Heidi Steendam at Monash University. This work was supported by the Australian Research Council's (ARC) Discovery funding schemes DP130101265 and DP150100003. alternative for situations where traditional RF communication is not possible due to safety reasons, e.g. in hospitals. Recently, VLC has also been considered for indoor positioning [2]-[12].

Since the development of GPS technology, the number of applications that make use of the user's position has exploded. However, indoors the reception of the satellite signals is reduced. Therefore, from the beginning of this century, researchers have tried to develop a positioning system for indoor areas. However, an accurate low-cost energy-efficient solution is still lacking. Compared to RF positioning solutions, visible light positioning (VLP) has a number of advantages. Firstly, luminaires are generally placed at regular intervals on the ceiling, so most positions in the room experience line-of-sight (LOS). Secondly, because light is blocked by opaque walls, no interference from adjacent rooms will be present. Thirdly, the deployment cost of the VLP solution is low, because lighting must be provided anyway, and the hardware cost of a white LED and a photodiode (PD) is negligible. Hence, VLP should be considered as a viable candidate for indoor localization.

Several approaches exist for positioning using VLP. Some already deployed solutions, e.g. ByteLight and Philips GE, use the CCD camera available in a mobile phone. Because of the large number of pixels, the CCD camera can extract accurate information about the direction of arrival of the light. However, the sensor quickly drains the battery of a mobile phone and because of its low refresh rate, the light should be modulated at a low frequency, resulting in flicker. Therefore, many recent papers consider the use of a PD, e.g. [3]-[12]. These papers apply several approaches. Some papers consider a direct estimation of the position e.g. [11], and others use a two-step approach by first extracting distance information based on the received signal strength (RSS) e.g. [3], [5], [7]-[9], time-(difference-)of-arrival (T(D)OA) e.g. [4], [10] or angle-of-arrival (AOA) e.g. [6], [12], and use trilateration or triangulation to obtain the user's position in the second step. The resulting position accuracy ranges between several centimetres and a metre, depending on how much position-related information is extracted from the light. Most researchers obtain position-related information by using directional LEDs (e.g. with higher Lambertian order) and a non-directional receiver, but other solutions considering a directional receiver combined with non-directional LEDs (e.g. with low Lambertian order) also exist. In the first approach, by narrowing the half-angle of the LED, the positioning accuracy can be improved within the LED's half-angle. However, when the half-angle is too narrow, many LEDs are required to obtain good positioning 
accuracy over an entire room. To overcome this limitation, recently researchers started investigating directional receivers. In [6], [12], the authors considered tilted PDs, mounted on the corners of a cube. Although accurate 3D positioning is possible with this approach, its practical use is limited as the resulting receiver is not compact. In this paper, we will consider a different directional receiver, i.e. the receiver structure from [13] consisting of PDs combined with apertures. The advantage of this receiver compared to [6], [12] is its compactness. The positions of the apertures are selected to obtain good angular diversity, i.e., the light captured by a receiving element will strongly depend on the direction of the light, implying the differences in RSS at the different PDs offer information on the direction of the light.

Although the existing papers on VLP promise good positioning accuracy, it is not clear to what extent the proposed algorithms are optimal. A way to assess the performance of algorithms is the Cramer-Rao bound (CRB), which is a theoretical lower bound on the mean-squared error (MSE) of an unbiased estimator. Only a few papers are available focussing on the CRB for VLP. In [14]-[15], the authors consider the CRB for directional LEDs and a non-directional receiver, based on RSS and AOA measurements, respectively, while [16] derives the CRB for a TOA approach. However, TOA requires accurate synchronization between the transmitted optical signals. In [17], we considered the receiver structure from [13], where we directly estimate the user's position based on the RSS values, and the CRB was derived for observations consisting of the time-average of the received signals. However, to distinguish between the time-averaged signals of the different LEDs, the LEDs must transmit signals that do not overlap in time, which will not only cause flicker, but also requires accurate time synchronization at the transmitters. Hence, the approach in [17] has its practical limitations.

In this paper, we use the same receiver structure as in [17], and consider the direct estimation of the position based on the RSS values. However, in contrast to [17], the RSS values are obtained by correlating the received signals with a number of reference signals related to the transmitted signals. By properly selecting the transmitted signals and the reference signals, we can distinguish between the different transmitted signals at the receiver, even if they are transmitted simultaneously. Hence, the LEDs may transmit continuously and no synchronization between the LEDs is required. In this paper, we derive the CRB for this approach, and evaluate the effect of different system parameters. We show that only a limited number of LEDs is required to achieve accurate positioning. Hence, as to obtain an illuminance of 500-700 lux in e.g. office spaces or supermarkets, a large number of LEDs is available, we only need a small subset of these luminaires for positioning purposes. This will reduce the installation cost of the positioning system. To assess the tightness of the CRB, which is derived for the case of a LOS link between the LEDs and the receiver only, we compare the results with the mean squared error (MSE) performance of the maximum likelihood (ML) estimator for the position, in the absence and presence of diffuse reflections of the light on walls and objects. We show that the MSE of the ML estimator closely matches the CRB and that if a diffuse component is present, the degradation of the MSE of the ML estimator only is substantial near the walls, indicating that the CRB is still a good lower bound if a diffuse component is present. In contrast to the ML estimator, where the performance must be evaluated through simulations because of the complex relationship between the receiver position and the received signal strengths, the CRB can be computed analytically with low complexity. Therefore the CRB derived in this paper is very useful for assessing and optimizing system performance. The approach in this paper outperforms the results from [17] and show that with realistic parameter settings, a position accuracy of a centimetre can be obtained.

\section{SYSTEM DESCRIPTION}

\section{A. Received Signals}

We consider the receiver introduced in [13], which contains $M$ receiving elements (RE). Each RE consists of a bare, circular PD with radius $R_{D}$ and an aperture, as shown in Fig. 1. The aperture is a circular hole with radius $R_{A}$ in an opaque screen that is located in a plane that is parallel to the plane of the PDs, and located at height $h_{A}$ above the plane of the PDs. We assume that the only light that reaches a PD is the light that passes through its aperture, and that the radius $R_{A}$ of the aperture is large compared to the wavelength of the light. As a result, the incident light will introduce a circular light $\operatorname{spot}^{1}$ on the plane of the PD (see Fig. 1b). In the following, we assume $R_{A}=R_{D}$, as in that case a different position of the receiver will always result in different RSS values. In contrast, when $R_{A} \neq R_{D}$, the dependency of the RSS values on the position may show ambiguities, reducing the positioning accuracy.

To the ceiling of the room are attached $K$ white LEDs at positions $\left(x_{S, i}, y_{S, i}\right), i=1, \ldots, K$, which can be modelled as generalized Lambertian LEDs with order $m_{i}$. We assume the plane of the receiver is parallel to the ceiling, at a distance $h$ below the ceiling, as shown in Fig. 2. Our goal is to estimate the position of $\left(x_{U}, y_{U}\right)$, which is a reference point on the receiver. In the system we consider, the apertures are symmetrically arranged around the reference point with the offset of the centre of the $j$ th aperture $\left(x_{A P, j}, y_{A P, j}\right)$ from the reference point given by $\left(\delta x_{j}, \delta y_{j}\right)$ so that $\left(x_{A P, j}, y_{A P, j}\right)=$ $\left(x_{U}+\delta x_{j}, y_{U}+\delta y_{j}\right)$. To achieve angular diversity, each PD is also offset from its aperture. As shown in Fig. 1b, the position of the PD is determined by the angle $\alpha_{A P, j}$ and the distance $d_{A P, j}$ between the centres of the aperture and the PD. The parameters $\left(\delta x_{j}, \delta y_{j}\right)$ and $\left(d_{A P, j}, \alpha_{A P, j}\right)$ depend on the receiver layout. We will show that the received signal strength can be written as a function of the incident and polar angles $\left(\phi_{j, i}, \alpha_{j, i}\right)$ between LED $i$ and RE $j$. Taking into account Fig. 2 , the relation between $\left(x_{S, i}, y_{S, i}\right)$ and $\left(x_{A P, j}, y_{A P, j}\right)$ is given

\footnotetext{
${ }^{1}$ The circular shape of the light spot is due to elementary geometry related to the intersection of elliptical cylinders and parallel planes. As the planes of the apertures and the PDs are parallel, the shapes and sizes of the intersections will be equal. Hence, as the aperture is circular with radius $R_{A}$, the light spot will also be circular with radius $R_{A}$.
} 


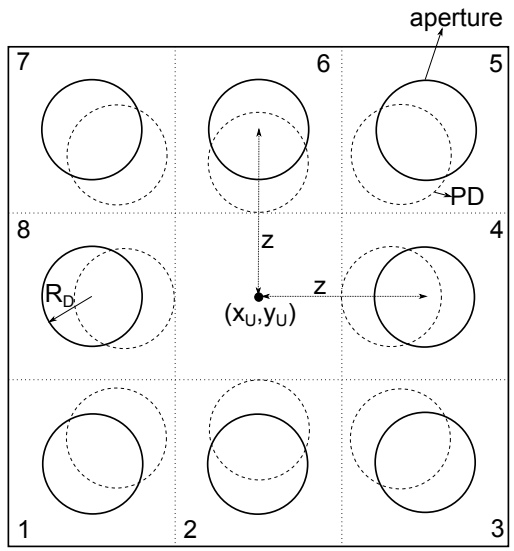

(a)

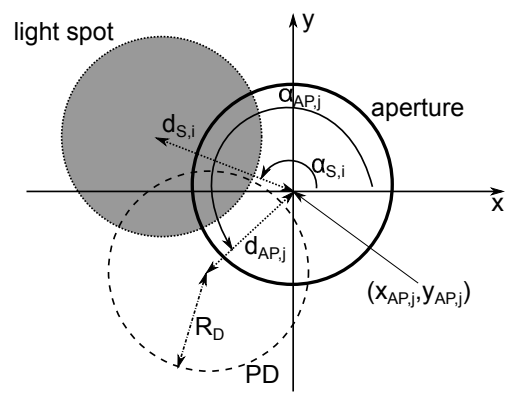

(b)

Fig. 1. a) Top view of a receiver structure consisting of $M=8$ REs and b) top view of one RE.

by

$$
\begin{aligned}
x_{S, i} & =x_{A P, j}+h \tan \phi_{j, i} \cos \alpha_{j, i} \\
y_{S, i} & =y_{A P, j}+h \tan \phi_{j, i} \sin \alpha_{j, i} .
\end{aligned}
$$

In the following, it will be shown that the RSS in a RE depends on the incident and polar angle. The angles $\left(\phi_{j, i}, \alpha_{j, i}\right)$ are deterministic functions of the positions of the apertures $\left(x_{A P, j}, y_{A P, j}\right)$ and the LEDs $\left(x_{S, i}, y_{S, i}\right)$ (1). The goal of this paper is to directly estimate the position $\left(x_{U}, y_{U}\right)$ of the receiver, while the relative distances $\left(\delta x_{j}, \delta y_{j}\right)$ of the apertures to the reference point on the receiver and the positions $\left(x_{S, i}, y_{S, i}\right)$ of the LEDs are known at the receiver. Hence, the angles $\left(\phi_{j, i}, \alpha_{j, i}\right)$ do not have to be determined by the receiver, but are only introduced to simplify the notations in the following.

The optical signal transmitted by LED $i$ equals $s_{i}(t)$. At RE $j$, the optical signal coming from the different LEDs is converted to an electrical signal $r_{j}(t)$ by the PD. This signal $r_{j}(t)$ consists of a linear combination of the transmitted signals $s_{i}(t)$. To separate the contributions from the different LEDs, the receiver correlates the signal $r_{j}(t)$ with a set of reference signals $\left\{\psi_{i}(t) \mid i=1, \ldots, K\right\}$ over the time interval $[0, T]$, where reference signal $\psi_{i}(t)$ is a function of the signal $s_{i}(t)$ transmitted by LED $i: r_{i}[j]=\int_{0}^{T} r_{j}(t) \psi_{i}(t) d t, j=1, \ldots, M$, $i=1, \ldots, K$. Defining the $M K \times 1$ vector $\mathbf{r}=\left(\mathbf{r}_{1}^{T} \ldots \mathbf{r}_{K}^{T}\right)^{T}$ as the vector of observations, with $\mathbf{r}_{\ell}=\left(r_{\ell}[1] \ldots r_{\ell}[M]\right)^{T}$,

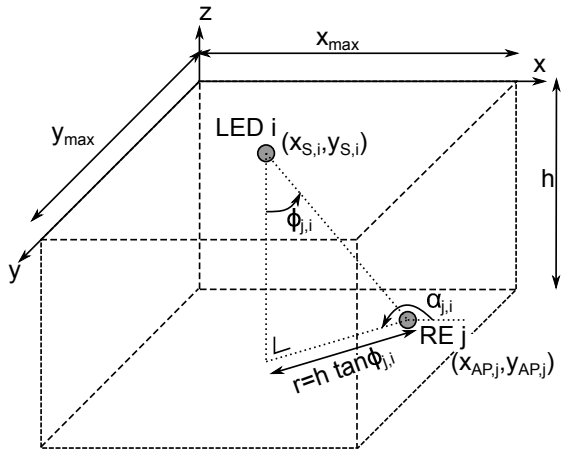

Fig. 2. The positions of LED $i$ and $\operatorname{RE} j$ in the room, and the definition of the incident angle $\phi_{j, i}$ and polar angle $\alpha_{j, i}$.

we obtain

$$
\mathbf{r}=R_{p} \tilde{\mathbf{H}} \boldsymbol{\mu}+\tilde{\mathbf{n}} .
$$

In (2), $R_{p}$ is the responsitivity of the PD and $\tilde{\mathbf{H}}$ is a $M K \times K^{2}$ matrix:

$$
\tilde{\mathbf{H}}=\left(\begin{array}{cccc}
\mathbf{H} & \mathbf{0} & \ldots & \mathbf{0} \\
\mathbf{0} & \mathbf{H} & \ldots & \mathbf{0} \\
\vdots & \vdots & & \vdots \\
\mathbf{0} & \mathbf{0} & \ldots & \mathbf{H}
\end{array}\right)
$$

with $(\mathbf{H})_{j, i}=h_{c}^{(j, i)}, j=1, \ldots, M, i=1, \ldots, K$ the $M \times K$ matrix of the channel gains. Further, the $K^{2} \times 1$ vector $\boldsymbol{\mu}$ is a function of the transmitted optical signals $s_{i}(t)$, $i=1, \ldots, K$, and is defined as $\boldsymbol{\mu}=\left(\boldsymbol{\mu}_{1}^{T} \ldots \boldsymbol{\mu}_{K}^{T}\right)^{T}$, with $\boldsymbol{\mu}_{\ell}=$ $\left(\mu_{\ell}[1] \ldots \mu_{\ell}[K]\right)^{T}$ and $\mu_{\ell}[i]=\int_{0}^{T} s_{i}(t) \psi_{\ell}(t) d t$. The $M K \times 1$ vector $\tilde{\mathbf{n}}=\left(\mathbf{n}_{1}^{T} \ldots \mathbf{n}_{K}^{T}\right)^{T}$, with $\mathbf{n}_{\ell}=\left(n_{\ell}[1] \ldots n_{\ell}[M]\right)^{T}$ is the contribution from the noise. The noise consists of shot noise and thermal noise. Assuming the bandwidth of the signals $s_{i}(t)$ is below $10 \mathrm{MHz}$, in many practical situations, the noise will be dominated by the shot noise [18]. Hence, in the following, we will only consider the contribution from the shot noise. The shot noise is dominated by signal-independent background light, so $\tilde{\mathbf{n}}$ can be modelled as zero-mean Gaussian random variables with covariance matrix $\frac{N_{0}}{2} \tilde{\mathbf{R}}$, where $\tilde{\mathbf{R}}=\mathbf{R}_{\psi} \otimes \mathbf{I}_{M}$, with $\otimes$ denoting the Kronecker product of the $K \times K$ matrix $\mathbf{R}_{\psi}$ and the $M \times M$ identity matrix $\mathbf{I}_{M}$, and $\left(\mathbf{R}_{\psi}\right)_{\ell, \ell^{\prime}}=\int_{0}^{T} \psi_{\ell}(t) \psi_{\ell^{\prime}}(t) d t$. Further, the noise level $N_{0}$ is defined as $N_{0}=2 q R_{p} p_{n} A_{D} \Delta \lambda$, where $q$ is the charge of an electron, $p_{n}$ is the background spectral irradiance, $A_{D}$ is the area of the PD and $\Delta \lambda$ is the bandwidth of the optical filter in front of the PD. Taking into account that the $i$ th LED is modelled as a generalized Lambertian LED with order $m_{i}$, the channel gain $h_{c}^{(j, i)}$ can be written as [18]

$$
h_{c}^{(j, i)}=\frac{m_{i}+1}{2 \pi h^{2}} A_{0}^{(j, i)} \cos ^{m_{i}+3} \phi_{j, i}
$$

where $A_{0}^{(j, i)}$ corresponds to the surface of the overlap area between PD $j$ and the light spot originating from LED $i$ :

$$
A_{0}^{(j, i)}=\left\{\begin{array}{cl}
2 R_{D}^{2} \arccos \left(\frac{d_{j, i}}{2 R_{D}}\right) & 0 \leq d_{j, i} \leq 2 R_{D} \\
-\frac{d_{j, i}}{2} \sqrt{4 R_{D}^{2}-d_{j, i}^{2}} & \\
0 & d_{j, i}>2 R_{D}
\end{array} .\right.
$$


This overlap area is determined by the distance $d_{j, i}$ between the centre of the light spot and the centre of the PD, which can be written as

$$
\begin{aligned}
d_{j, i} & =\left[\left(d_{S}^{(j, i)} \cos \alpha_{S}^{(j, i)}-d_{A P, j} \cos \alpha_{A P, j}\right)^{2}\right. \\
& \left.+\left(d_{S}^{(j, i)} \sin \alpha_{S}^{(j, i)}-d_{A P, j} \sin \alpha_{A P, j}\right)^{2}\right]^{1 / 2} \\
& =\left[\left(\frac{h_{A}}{h}\left(x_{S, i}-x_{A P_{j}}\right)-d_{A P, j} \cos \alpha_{A P, j}\right)^{2}\right. \\
& \left.+\left(\frac{h_{A}}{h}\left(y_{S, i}-y_{A P_{j}}\right)-d_{A P, j} \sin \alpha_{A P, j}\right)^{2}\right]^{1 / 2}
\end{aligned}
$$

The angle $\alpha_{S}^{(j, i)}$ and position $d_{S}^{(j, i)}$ of the light spot are determined by the angle-of-incidence $\phi_{j, i}$ and polar angle $\alpha_{j, i}$ of the light arriving from LED $j$ on PD $i: d_{S}^{(j, i)}=h_{A} \tan \phi_{j, i}$ and $\alpha_{S}^{(j, i)}=\pi+\alpha_{j, i}$. Further, the second line in (6) is obtained by substituting (1).

\section{B. Cramer-Rao Bound}

The mean-squared error (MSE) of any unbiased estimate $\hat{\boldsymbol{\theta}}=\left(\hat{x}_{U}, \hat{y}_{U}\right)$ of the position $\boldsymbol{\theta}=\left(x_{U}, y_{U}\right)$ based on the observation (2) is lower bounded by the CRB, defined as

$$
\mathrm{MSE}=E\left[\left(x_{U}-\hat{x}_{U}\right)^{2}+\left(y_{U}-\hat{y}_{U}\right)^{2}\right] \geq \operatorname{trace}\left(\mathbf{F}_{U}^{-1}\right) .
$$

In (7), $\mathbf{F}_{U}$ is the Fisher information matrix (FIM) [19]:

$$
\mathbf{F}_{U}=E\left[\left(\boldsymbol{\nabla}_{\theta} \ln p(\mathbf{r} \mid \boldsymbol{\theta})\right)\left(\boldsymbol{\nabla}_{\theta} \ln p(\mathbf{r} \mid \boldsymbol{\theta})\right)^{T}\right] .
$$

Bearing in mind that $\mathbf{r} \mid \boldsymbol{\theta} \sim N\left(R_{p} \tilde{\mathbf{H}} \boldsymbol{\mu}, \frac{N_{0}}{2} \tilde{\mathbf{R}}\right)$, it is shown in the Appendix that

$$
\mathbf{F}_{U}=\frac{2 R_{p}^{2}}{N_{0}}\left[\begin{array}{ll}
Z^{x_{U} x_{U}} & Z^{x_{U} y_{U}} \\
Z^{y_{U} x_{U}} & Z^{y_{U} y_{U}}
\end{array}\right] .
$$

In (9), $Z^{a b}, a, b \in\left\{x_{U}, y_{U}\right\}$ is defined as

$$
Z^{a b}=\operatorname{trace}\left(\mathbf{R}_{\psi}^{-1} \mathbf{W}^{a b}\right),
$$

where $\left(\mathbf{W}^{a b}\right)_{\ell, \ell^{\prime}}=\boldsymbol{\mu}_{\ell}^{T} \mathbf{X}^{a b} \boldsymbol{\mu}_{\ell^{\prime}}$ and

$$
\begin{aligned}
\left(\mathbf{X}^{a b}\right)_{i, i^{\prime}} & =\left[\left(\frac{\partial}{\partial a} \mathbf{H}\right)^{T}\left(\frac{\partial}{\partial b} \mathbf{H}\right)\right]_{i, i^{\prime}} \\
& =\sum_{j=1}^{M} \frac{\partial}{\partial a} h_{c}^{(j, i)} \frac{\partial}{\partial b} h_{c}^{\left(j, i^{\prime}\right)} .
\end{aligned}
$$

In (11), it can be observed that in order to compute the $\mathrm{CRB}$, we need the derivative of the channel gain $h_{c}^{(j, i)}$ with respect to $x_{U}$ and $y_{U}$ :

$$
\begin{array}{r}
\frac{\partial}{\partial a} h_{c}^{(j, i)=\frac{m_{i}+1}{2 \pi h^{2}}[}\left[\left(\frac{\partial}{\partial z} A_{0}^{(j, i)}\right) \cos ^{m_{i}+3} \phi_{j, i}\right. \\
\left.+A_{0}^{(j, i)} \frac{\partial}{\partial z} \cos ^{m_{i}+3} \phi_{j, i}\right]
\end{array}
$$

with $a \in\left\{x_{U}, y_{U}\right\}$. Taking into account (1), (5) and (6), it follows that

$$
\begin{aligned}
\frac{\partial}{\partial x_{U}} \cos ^{m_{i}+3} \phi_{j, i} & =\frac{m_{i}+3}{h^{2}}\left(x_{S, i}-x_{A P, j}\right) \cos ^{m_{i}+5} \phi_{j, i} \\
\frac{\partial}{\partial y_{U}} \cos ^{m_{i}+3} \phi_{j, i} & =\frac{m_{i}+3}{h^{2}}\left(y_{S, i}-y_{A P, j}\right) \cos ^{m_{i}+5} \phi_{j, i} .
\end{aligned}
$$

For $0 \leq d_{j, i} \leq 2 R_{D}$ and $a \in\left\{x_{U}, y_{U}\right\}, \frac{\partial}{\partial a} A_{0}^{(j, i)}$ yields

$$
\frac{\partial}{\partial a} A_{0}^{(j, i)}=-\sqrt{4 R_{D}^{2}-d_{j, i}^{2}} \frac{\partial}{\partial a} d_{j, i}
$$

and

$$
\begin{array}{r}
\frac{\partial}{\partial x_{U}} d_{j, i}=-\frac{R_{D}}{h d_{j, i}}\left(\frac{h_{A}}{h}\left(x_{S, i}-x_{A P, j}\right)\right. \\
\left.+d_{A P, j} \cos \alpha_{A P, j}\right) \\
\frac{\partial}{\partial y_{U}} d_{j, i}=-\frac{R_{D}}{h d_{j, i}}\left(\frac{h_{A}}{h}\left(y_{S, i}-y_{A P, j}\right)\right. \\
\left.+d_{A P, j} \sin \alpha_{A P, j}\right) .
\end{array}
$$

\section{Transmitted Optical Signals}

The CRB depends on the transmitted optical signals $s_{i}(t)$ and reference signals $\psi_{\ell}(t)$ through the vector $\boldsymbol{\mu}_{\ell}$ and the matrix $\mathbf{R}_{\psi}$. To assess the effect of $s_{i}(t)$ and $\psi_{\ell}(t)$, we consider the following transmitted optical signals $s_{i}(t)$. Taking into account that the transmitted optical signals must be real-valued and positive, we assume the transmitted optical signal is a dcbiased windowed sinusoid waveform with duration $T$ :

$$
s_{i}(t)=A_{i} w(t)\left(1+\cos \left(2 \pi f_{c, i} t\right)\right)
$$

where the window function $w(t)$ has no contributions outside the interval $[0, T]: w(t)=0$ for $t \notin[0, T]$. We denote $A_{i} w(t)$ the dc component and $A_{i} w(t) \cos \left(2 \pi f_{c, i} t\right)$ the carrier component of the signal. Further, the frequency $f_{c, i}$ of the sinusoid is assumed to have an integer number of periods within the interval $[0, T]$, and the frequencies corresponding to the different LEDs are orthogonal over $[0, T]$, i.e. $\left(f_{c, i}-f_{c, i^{\prime}}\right) T$ is integer for $i, i^{\prime}=1, \ldots, K$. The selection of the frequencies can be based on the discrete Fourier transform, resulting in orthogonal frequency division multiplexing (OFDM), which is commonly considered for optical wireless communication [20]-[23]. The window-function is a smooth function, e.g. a raised cosine window:

$$
w(t)=\left(1+\cos \left(\frac{2 \pi}{T}\left(t-\frac{T}{2}\right)\right)\right) .
$$

Further, the window function $w(t)$ is assumed to have a narrow bandwidth compared to the frequencies $f_{c, i}$ of the sinusoids. Taking into account (16) and (17), the optical power transmitted by LED $i$ equals:

$$
P_{o p t, i}=\frac{1}{T} \int_{0}^{T} s_{i}(t) d t=A_{i} .
$$

For the reference signals $\psi_{\ell}(t)$, we consider two different cases. In the first case, we select reference signal $i$ to be the entire transmitted optical signal transmitted by LED $i$, i.e. $\psi_{i}(t)=s_{i}(t)$. In that case, it can be verified that $\mathbf{R}_{\psi}=\left(\boldsymbol{\mu}_{1} \ldots \boldsymbol{\mu}_{K}\right)$. As a result, the matrix $\mathbf{W}^{a b}$ can be rewritten as $\mathbf{W}^{a b}=\mathbf{R}_{\psi}^{T} \mathbf{X}^{a b} \mathbf{R}_{\psi}$, so that the elements $Z^{a b}$ (10) reduce to $Z^{a b}=\operatorname{trace}\left(\mathbf{R}_{\psi} \mathbf{X}^{a b}\right)$, where we used $\mathbf{R}_{\psi}^{T}=\mathbf{R}_{\psi}$. By substituting (16) and (17) in $\mathbf{R}_{\psi}$, we obtain

$$
Z_{1}^{a b}=\operatorname{trace}\left(\mathbf{R}_{s, 1} \mathbf{X}^{a b}\right)
$$


where $\mathbf{R}_{s, 1}=\frac{3 T}{2}\left(\mathbf{A}^{T} \mathbf{A}+\frac{1}{2} \operatorname{diag}(\mathbf{A} \circ \mathbf{A})\right)$, with $\mathbf{A}=$ $\left(A_{1} \ldots A_{K}\right)$ is the vector of transmitted optical powers, o denotes the Hadamard product, and $\operatorname{diag}(\mathbf{A})$ is the diagonal matrix with as diagonal elements the elements of $\mathbf{A} \circ \mathbf{A}$.

As can be observed, by correlating the received signal $r_{j}(t)$ with the entire transmitted optical signals $s_{i}(t)$, the different components of the resulting observation are correlated, i.e. $\mathbf{R}_{\psi}=\mathbf{R}_{s, 1}$ is not a diagonal matrix. This correlation is due to the presence of the dc component of $s_{i}(t)$ in the reference signals. As this correlation will make it harder to separate the contributions from the different LEDs, we consider the second case, where the reference signal $\psi_{i}(t)$ does not contain the dc component from $s_{i}(t): \psi_{i}(t)=A_{i} w(t) \cos \left(2 \pi f_{c, i} t\right)$, $i=1, \ldots, K$. In that case, $\boldsymbol{\mu}_{\ell}$ and $\mathbf{R}_{\psi}$ reduce to

$$
\begin{aligned}
\boldsymbol{\mu}_{\ell}[i] & =\frac{3 T}{4} A_{i} A_{\ell} \delta_{i, \ell} \\
\mathbf{R}_{\psi} & =\frac{3 T}{4} \operatorname{diag}(\mathbf{A} \circ \mathbf{A}) .
\end{aligned}
$$

Inspecting (20) reveals that in this case, again $\mathbf{R}_{\psi}=$ $\left(\boldsymbol{\mu}_{1} \ldots \boldsymbol{\mu}_{K}\right)$, so that $Z^{a b}$ can be rewritten as

$$
Z_{2}^{a b}=\operatorname{trace}\left(\mathbf{R}_{s, 2} \mathbf{X}^{a b}\right),
$$

where $\mathbf{R}_{s, 2}=\mathbf{R}_{\psi}=\frac{3 T}{4} \operatorname{diag}(\mathbf{A} \circ \mathbf{A})$.

\section{NUMERICAL RESULTS}

In the evaluation of the CRB, we consider the receiver structure shown in Fig. 1a. The receiver consists of $M=8$ REs, where the relative positions $\left(\delta x_{j}, \delta y_{j}\right)$ of the apertures with respect to the position $\left(x_{U}, y_{U}\right)$ of the receiver are given by

$$
\begin{aligned}
\delta x_{j} & =\epsilon_{x, j} \cos \chi-\epsilon_{y, j} \sin \chi \\
\delta y_{j} & =\epsilon_{x, j} \sin \chi+\epsilon_{y, j} \cos \chi
\end{aligned}
$$

where $\boldsymbol{\epsilon}_{x}=\epsilon R_{D}\left(\begin{array}{llllllll}-1 & 0 & 1 & 1 & 1 & 0 & -1 & -1\end{array}\right)$ and $\boldsymbol{\epsilon}_{y}=$ $\epsilon R_{D}(-1-1-10101110)$. The angle $\chi$ in (22) expresses the orientation of the receiver in the $(x, y)$-plane, while the parameter $\epsilon$ determines the distance between the apertures and the central point $\left(x_{U}, y_{U}\right)$ of the receiver. In this paper, we restrict our attention to the case where the receiver is positioned in the $(x, y)$-plane, i.e., the plane parallel to the ceiling where the LEDs are located ${ }^{2}$. Further, the PDs are placed at positions $d_{A P, j}=\zeta R_{D}$ and $\alpha_{A P, j}=j \frac{\pi}{4}$ relative to their apertures, where $\zeta$ determines the field-of-view of the receiver and the angles $\alpha_{A P, j}$ are selected to offer good angular diversity [13].

Unless stated otherwise, the $K=P^{2}$ LEDs are uniformly placed on the ceiling according to a rectangular pattern at

\footnotetext{
${ }^{2} \mathrm{~A}$ tilt of the receiver will have a deterministic effect on the RSS values. In mobile devices, inertial measurement units (IMUs) are available such as an accelerometer, gyroscope and compass. These IMUs are able to accurately determine the orientation of the mobile device. Hence, the orientation of the receiver is prior known, and can be incorporated in the positioning process. In [24], the authors used this prior knowledge on the tilt to compensate it and investigate its effect on the positioning performance. Their conclusion is that after compensation of the tilt, the performance degradation is marginal. As a tilt in our system has a similar effect on the RSS values compared to [24], we can extrapolate the results from [24] to our system and conclude that the orientation of the receiver will have a marginal effect on the positioning performance only, as long as the REs have a LOS connection with the LEDs.
}

the positions $x_{S}(i)=\frac{x_{\max }}{2 P}+(i-1)_{P} \frac{x_{\max }}{P}$ and $y_{S}(i)=$ $\frac{y_{\max }}{2 P}+\left\lfloor\frac{i-1}{P}\right\rfloor \frac{y_{\max }}{P}, i=1, \ldots, K$, where $x_{\max }$ and $y_{\max }$ are the length and width of the considered area, $(x)_{P}$ is the modulo- $P$ operation of $x$, and $\lfloor x\rfloor$ is the largest integer not exceeding $x$. Unless mentioned otherwise, we assume the LEDs have a non-directional radiation pattern, i.e. they can be modelled as Lambertian with order $m_{i}=1$. Further, we assume the ceiling is at a height $h=2 \mathrm{~m}$ above the receiver. In order to determine the shot noise power spectral density $N_{0}=2 q R_{p} p_{n} A_{D} \Delta \lambda$, we assume a background spectral irradiance $p_{n}=5.8 \times 10^{-6} \mathrm{~W} / \mathrm{cm}^{2} \cdot \mathrm{nm}$, a value that is commonly used in the literature [18]. Further, the PD has a radius $R_{D}=1 \mathrm{~mm}$ and responsitivity $R_{p}=0.4 \mathrm{~mA} / \mathrm{mW}$ [25]. The optical filter passes only visible light frequencies corresponding to 380 to $740 \mathrm{~nm}$, resulting in an optical bandwidth of $\Delta \lambda=360 \mathrm{~nm}$. The resulting noise spectral density equals $N_{0}=8.4 \times 10^{-24} \mathrm{~A}^{2} / \mathrm{Hz}$. We assume the LEDs have the same transmit power (18): $P_{o p t, i}=A_{i}=A, i=1, \ldots, K$. In that case, $\mathbf{A}^{T} \mathbf{A}=A^{2} \mathbf{E}_{K}$ and $\mathbf{A} \circ \mathbf{A}=A^{2} \mathbf{I}_{K}$, where $\mathbf{E}_{K}$ is the $K \times K$ matrix of all ones, and $\mathbf{I}_{K}$ is the $K \times K$ identity matrix. Inspecting (9), (19) and (21), it can be observed that the FIM is proportional to the ratio $\gamma$ :

$$
\gamma \triangleq 2 T A^{2} R_{p}^{2} / N_{0}
$$

which is related to the signal-to-noise ratio ${ }^{3}$. Hence, the CRB will be inversely proportional to the length $T$. In the following, we assume the transmitted optical power of the non-directional LEDs with Lambertian order $m_{i}=1$ equals $A=1 \mathrm{~W}$.

We first evaluate the square root of the CRB (rCRB) as function of the position $\left(x_{U}, y_{U}\right)$ of the receiver. In Fig. 3, the $\mathrm{rCRB}$ is shown for the two cases from Section II-C, for $K=4,9$ and 16 and $10 \log _{10} \gamma=195.81 \mathrm{~dB}$, corresponding to a time window $T=1 \mathrm{~ms}$. We also show the rCRB from [17]. As expected, the value of the rCRB depends on the position of the receiver. In a large part of the considered $10 \mathrm{~m}$ $\times 10 \mathrm{~m}$ area, the $\mathrm{rCRB}$ is relatively constant, but near the edges of the area, the rCRB increases, for all three cases. This is expected, as near the edges of the area, the receiver captures less light compared to the middle of the area, due to the directionality of the REs in the receiver [13] and the radiation pattern of the LEDs. Comparing the spatial average of the $\mathrm{rCRB}\left(\mathrm{rCRB}_{\mathrm{av}}\right)$ for the three cases, i.e. the average over all positions in the area, we observe that Case 1 offers the highest accuracy, followed by Case 2 and the rCRB from [17] gives the lowest accuracy. This can be explained as in Case 1 , the received signal $r_{j}(t)$ is correlated with the entire transmitted signal $s_{i}(t)$, while in Case 2, by correlating with the carrier component only, we remove the dc-components from the received signal. Similarly, for the approach from [17], where the received signal $r_{j}(t)$ is time-averaged over the interval $[0, T]$, we remove the carrier components from the signal and keep only the dc-components of the signal. Hence, Case 1 uses most of the information. Comparing the three cases, however, reveals that the reduction of the accuracy

\footnotetext{
${ }^{3}$ The signal-to-noise ratio is defined as the ratio of the received useful power to the received noise power, i.e. $\mathrm{SNR}=2 T A^{2}\left(h_{c}^{(j, i)}\right)^{2} R_{p}^{2} / N_{0}=\gamma\left(h_{c}^{(j, i)}\right)^{2}$
} 

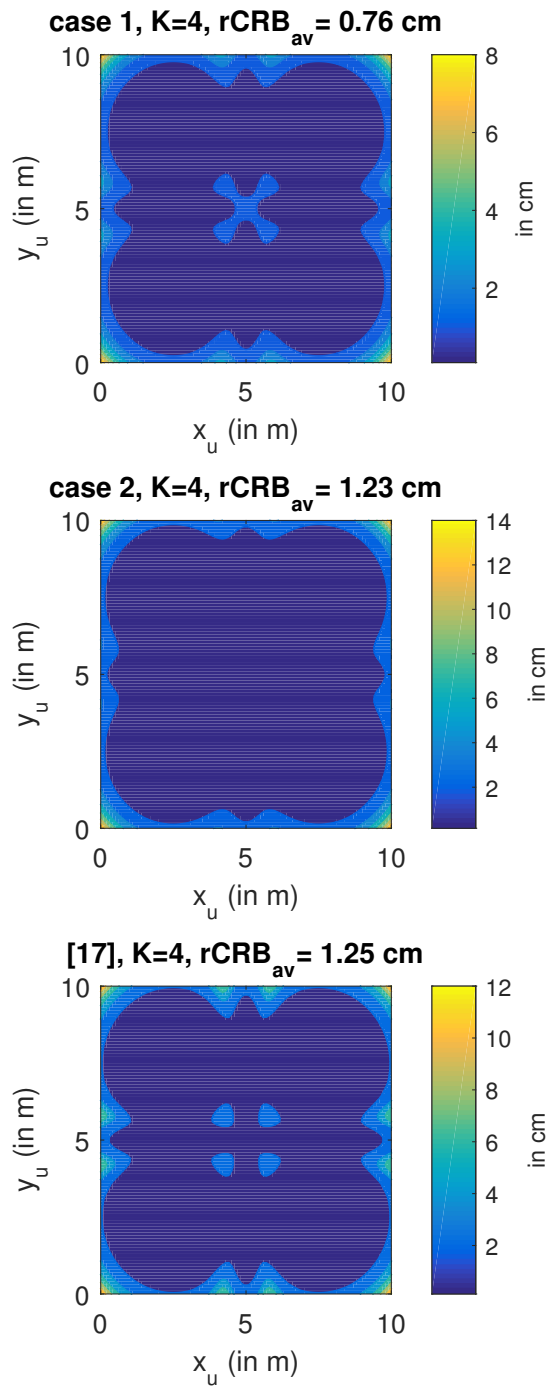
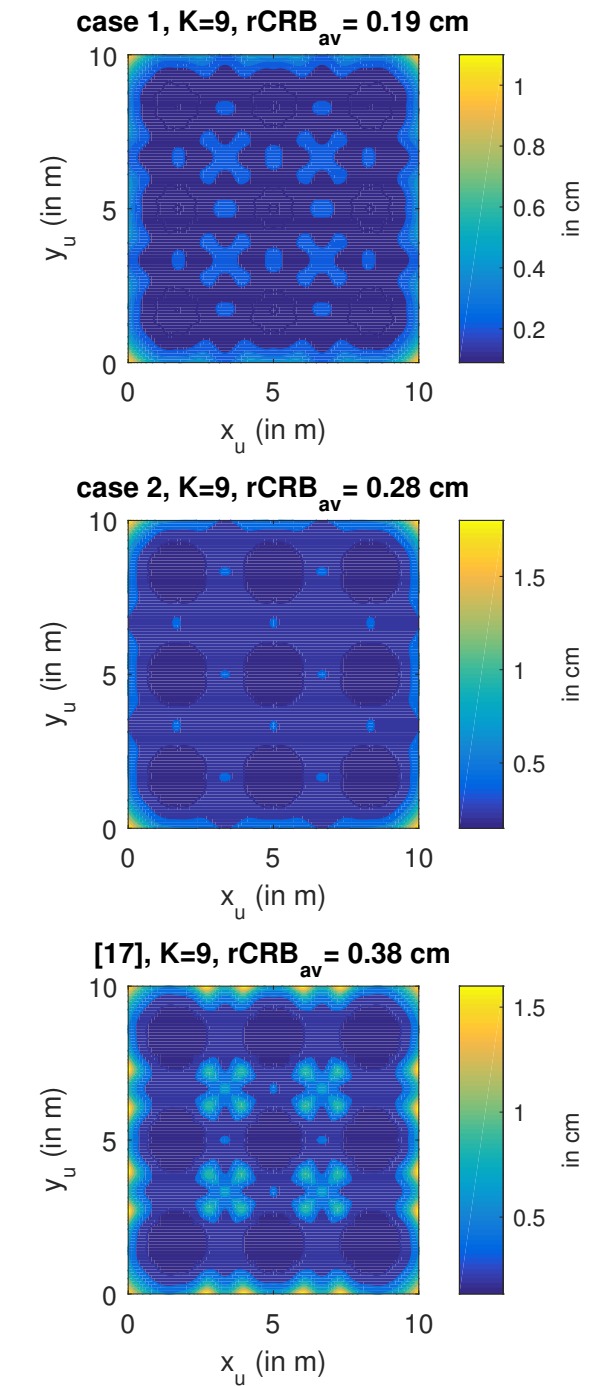
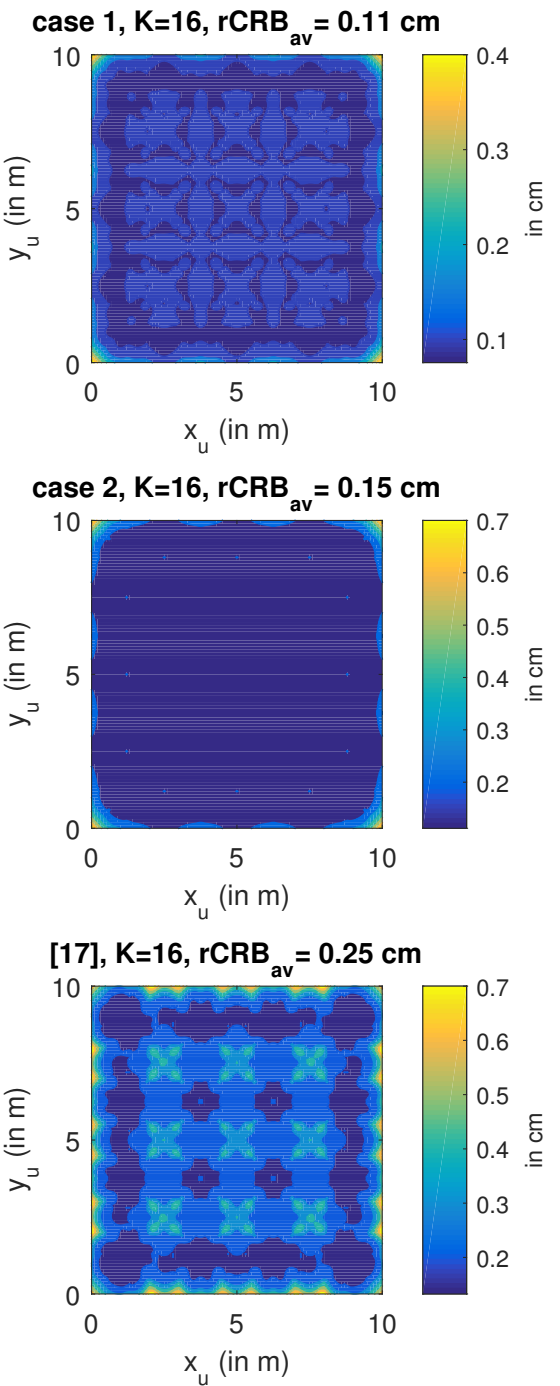

Fig. 3. Square root of the CRB as function of the position $\left(x_{U}, y_{U}\right)$ of the receiver in the room, for $10 \log _{10} \gamma=195.81 \mathrm{~dB}, x_{\mathrm{max}}=y_{\mathrm{max}}=10 \mathrm{~m}$, $\epsilon=5, h_{A}=R_{D}, \chi=0^{\circ}$ and $\zeta=0.5$.

is moderate. Further, in the figure, we observe that the average rCRB decreases for an increasing number of LEDs.

Next, we evaluate the influence of the receiver layout and the orientation of the receiver. Our simulations showed that neither the relative distance $\epsilon$ of the apertures w.r.t. the centre of the receiver, nor the orientation $\chi$ of the receiver have a noticeable influence on the performance. The independence of the performance to $\epsilon$ suggests that other configurations of the REs, e.g. by placing the REs on a line, will not affect the positioning ability. Further, the independence of the rCRB to the orientation $\chi$ can be attributed to the angular symmetry of the relative positions of the PDs with respect to their apertures, i.e., the angles $\alpha_{A P, j}$ are uniformly distributed over the interval $[0,2 \pi]$. However, the performance will strongly depend on the relative distance $\zeta R_{D}$ of the PDs w.r.t. their apertures, as this influences both the angular diversity and the field-of-view of the receiver, as well as the area of the PD, as this determines the amount of light captured by the receiver. First, we look at the effect of $\zeta$. In Fig. 4, we show the spatial average of the $\mathrm{rCRB}$ as function of $\zeta$. When $\zeta$ is

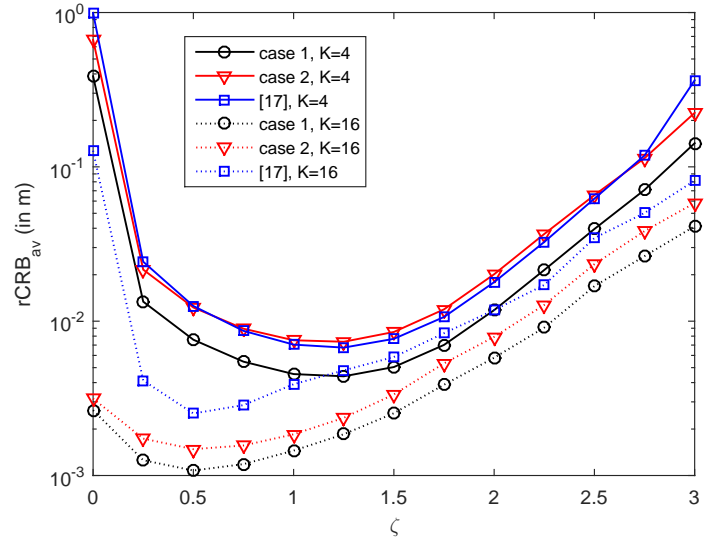

Fig. 4. Spatial average of $\mathrm{rCRB}$ as function of the relative distance $\zeta$ of the PDs w.r.t. their apertures, for $10 \log _{10} \gamma=195.81 \mathrm{~dB}, \epsilon=5, h_{A}=R_{D}$, $\chi=0^{\circ}$ and $x_{\max }=y_{\max }=10 \mathrm{~m}$. 
small, all REs will approximately receive the same amount of light, independent of the position of the receiver, i.e., although the receiver has the largest field-of-view, the angular diversity in the receiver is small. Hence, minimal information can be obtained about the polar angle $\alpha_{j, i}$, resulting in a lower accuracy. By increasing $\zeta$, the receiver will exhibit a larger angular diversity, i.e., the light captured by a RE strongly depends on the direction of the light. Moreover, by increasing $\zeta$, the receiver can detect larger incident angles, so that positions far away can better be determined. However, this comes at the cost of a slightly reduced field-of-view, as for small incident angles less light will be captured by the REs, resulting in a deterioration of the accuracy. The optimum value of $\zeta$ is in the range $\zeta \in[0.5,1.5]$. This optimum value reduces when the number of LEDs increases. This can be explained as follows. When $K$ is small, despite the uniform placement of the LEDs at the ceiling, the incident angle $\phi_{j, i}$ for the edges of the area is relatively large. Hence, to receive sufficient light for positioning at these positions, $\zeta$ must be sufficiently large. When $K$ is large, the distance between the receiver and the nearest LED becomes smaller, resulting in a smaller incident angle, even near the edges of the area. Hence, the value of $\zeta$ can reduce. Secondly, we evaluate the effect of the height $h_{A}$ on the average of the rCRB over all positions in a $10 \mathrm{~m} \times$ $10 \mathrm{~m}$ area, assuming the relative positions of the photodiodes with respect to their apertures are fixed, i.e. $\zeta$ is fixed. As can be observed in Fig. 5, the bound increases as function of $h_{A}$. This can be explained as follows. By changing the height $h_{A}$, the field-of-view of the receiver alters. For decreasing $h_{A}$, the field-of-view will become larger, so that, especially for positions at the edges of the area, the bound improves as more light will be received by the photodiodes. However, this effect is moderate: decreasing $h_{A}$ will only result in a slight improvement of the bound. When $h_{A}>R_{D}$, the bound increases significantly due to the reduction of the field-of-view. When $h_{A}$ is too large $\left(h_{A}>1.3 R_{D}\right.$ in Fig. 5), positions near the edges of the area will receive insufficient light to accurately determine their position because of this smaller field-of-view, resulting in an ill-conditioned FIM (9). Although the reduction of the field-of-view can be counteracted by increasing the distance between the photodiodes and their apertures, this will result in a larger receiver. For a compact receiver, the distance $h_{A}$ therefore must be $h_{A} \leq R_{D}$. Thirdly, we investigate the effect of the area of the PD. The dependency of the spatial average of rCRB on the radius $R_{D}$ of the PD is shown in Fig. 6. The rCRB is inversely proportional to $R_{D}$, or the square root of the PD area. Inspecting the elements of the FIM (9), we see that both the noise level $N_{0}$ and the channel gain $h_{c}^{(j, i)}$ are proportional to $R_{D}^{2}$, the latter through the area of overlap $A_{0}^{(j, i)}$ (5) between the light spot and the PD. Hence, the elements of the FIM are proportional to $R_{D}^{2}$. Taking into account the results from Figs. $4-6$, we can conclude that excellent positioning accuracy can be obtained in a $10 \mathrm{~m} \times 10 \mathrm{~m}$ area with only four $1 \mathrm{~W}$ LEDs using a compact receiver.

In Fig. 7 we study the rCRB when the spacing between the LEDs changes. To this end, we consider a $10 \mathrm{~m} \times 10 \mathrm{~m}$ area, where four $1 \mathrm{~W}$ LEDs are placed in a square grid with edge

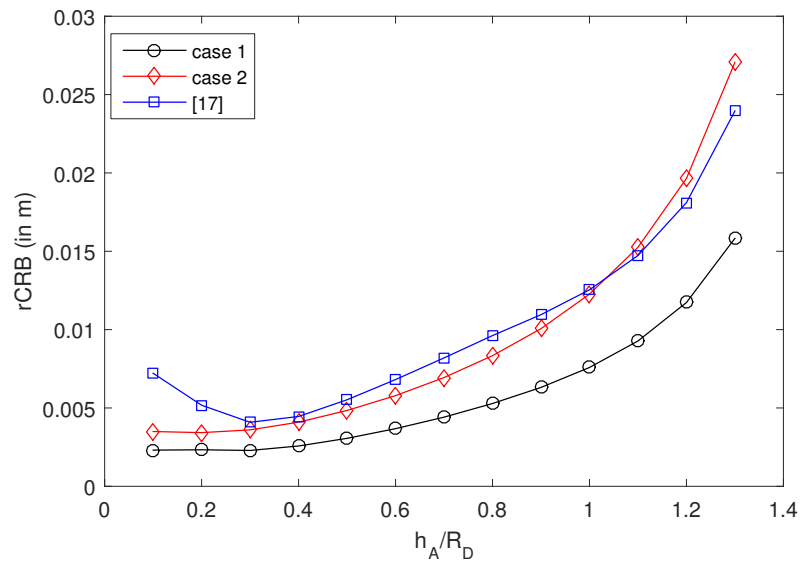

Fig. 5. Influence of the height $h_{A}$ on $\mathrm{rCRB}_{\mathrm{av}}, 10 \log _{10} \gamma=195.81 \mathrm{~dB}$, $K=4, x_{\max }=y_{\max }=10 \mathrm{~m}$ and $\zeta=0.5$.

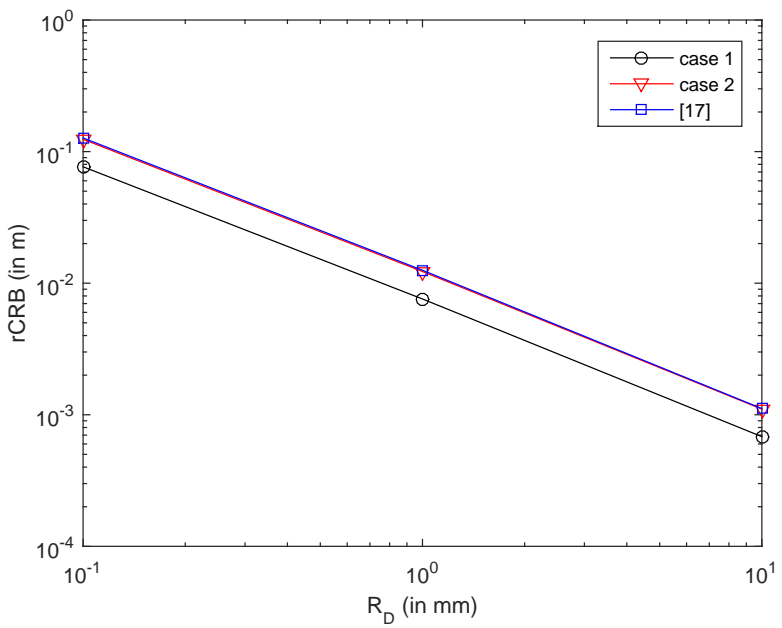

Fig. 6. Influence of the area of the PD on $\mathrm{rCRB}_{\mathrm{av}}, 10 \log _{10} \gamma / R_{D}^{2}=$ $255.81 \mathrm{~dB} \mathrm{~m}^{-2}, K=4, x_{\max }=y_{\max }=10 \mathrm{~m}, h_{A}=R_{D}$ and $\zeta=0.5$.

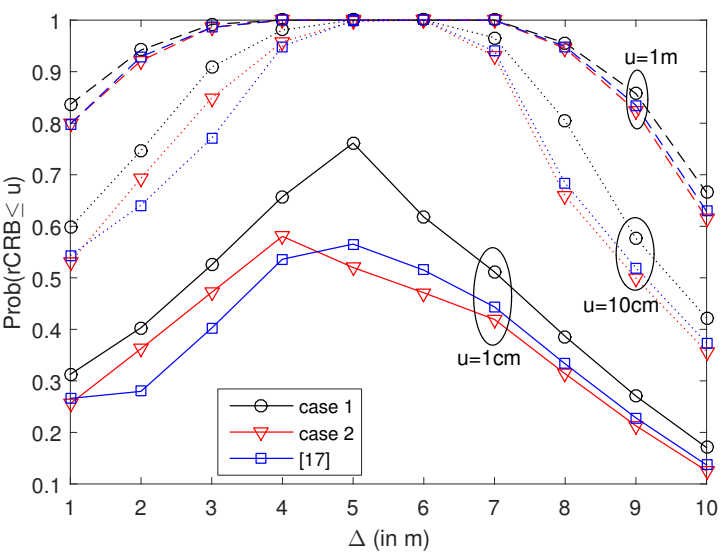

Fig. 7. Influence of the spacing of the LEDs on rCRB, $10 \log _{10} \gamma=$ $195.81 \mathrm{~dB}, K=4$ and $\zeta=0.5$. 
side $\Delta$, i.e. the LEDs have positions $(5 \mathrm{~m}-\Delta / 2,5 \mathrm{~m}-\Delta / 2)$, $(5 \mathrm{~m}-\Delta / 2,5 \mathrm{~m}+\Delta / 2),(5 \mathrm{~m}+\Delta / 2,5 \mathrm{~m}-\Delta / 2)$ and $(5 \mathrm{~m}+\Delta / 2,5 \mathrm{~m}+\Delta / 2)$. We show in the figure the probability of the rCRB being smaller than $u$ for different spacings $\Delta$. As can be observed, the best performance is obtained when the spacing is around $5 \mathrm{~m}$, i.e. when the LEDs are uniformly distributed over the area. This can be explained as follows. When the spacing is small, not only the distance between the LEDs and the positions at the boundaries of the considered area grows, but also the incident angles are relatively large. As a consequence, the received signal strengths for these positions will reduce resulting in a worse performance. On the other hand, when the spacing between the LEDs is large, e.g. when the LEDs are placed in the corners of the area, the positions in the center of the area will have lower performance, because of the large distance to the LEDs and the large incident angles. Hence, we can conclude that the best positioning performance will occur when the LEDs are distributed as uniformly as possible over the area. Further, we can conclude that centimetre accuracy can be obtained if one $1 \mathrm{~W}$ LED per $25 \mathrm{~m}^{2}$ is used for positioning.

Until now, we concentrated on the case where nondirectional LEDs are used for positioning. Although most light sources for illumination are non-directional, corresponding to a Lambertian order $m_{i}=1$, in some situations directional light sources with higher Lambertian order are used. Hence, we look at the effect of the directionality of the source. In Fig. 8, the probability of the rCRB being smaller than $u$ is shown as function of $u$ for two cases. In the first case, four non-directional $A=1 \mathrm{~W}$ LEDs with $m_{i}=1$ are attached to the ceiling of a $10 \mathrm{~m} \times 10 \mathrm{~m}$ area, whereas in the second case, directional LEDs with $m_{i}=5$ are used. To allow for a fair comparison, we consider the case where the illuminance of the area is the same for both cases. Taking into account that for $m_{i}=1$, the semi-angle of the LED is 60 degrees, whereas for $m_{i}=5$, this semi-angle is 30 degrees, we need 36 directional $A=1 / 9 \mathrm{~W}$ LEDs to have roughly the same illuminance. As can be observed in the figure, the performance is improved in the case of the directional LEDs, and the resulting positioning accuracy is also more uniform over the area, i.e. the curves for $m_{i}=5$ are steeper than for $m_{i}=1$. This performance improvement can be explained by the reduction of the relative distance between a position in the area and the LEDs, and the presence of more signals to determine the position, although the optical power per LED is reduced. We can conclude that, the more uniformly spaced LEDs we use for positioning, the more uniform the positioning error over the area will be.

Next, the influence of the duration $T$ of the correlation is shown in Fig. 9. The figure shows the probability of the rCRB being smaller than $u$, assuming four $1 \mathrm{~W}$ LEDS are used for positioning in a $10 \mathrm{~m} \times 10 \mathrm{~m}$ area. Using a $T=1 \mathrm{~ms}$ interval, more than half of the positions exhibits a position accuracy of better than $1 \mathrm{~cm}$, and in all positions the error is smaller than $10 \mathrm{~cm}$. By increasing the correlation interval to $10 \mathrm{~ms}$, we can increase the accuracy so that even in more than $90 \%$ of the positions, an error smaller than $1 \mathrm{~cm}$ can be obtained. Hence, taking into account the excellent accuracy corresponding to a short correlation time, we conclude that with our approach,

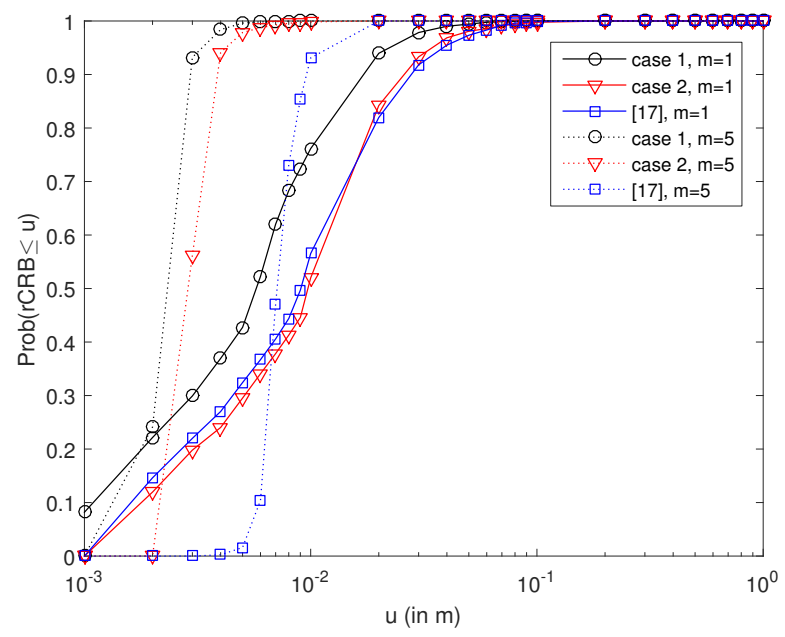

Fig. 8. Influence of the Lambertian order of the LEDs, $10 \log _{10} \gamma / A^{2}=$ $195.81 \mathrm{~dB}, \zeta=0.5$.

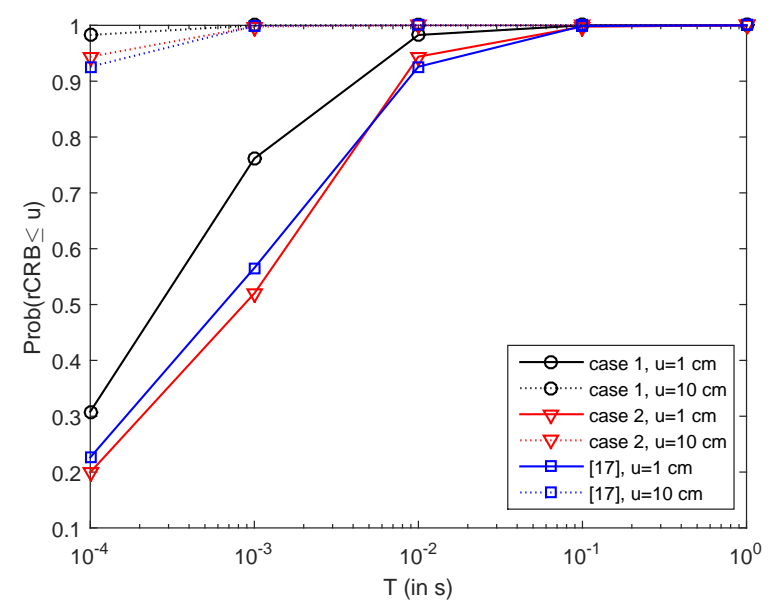

Fig. 9. Influence of the correlation time $T$ on $\mathrm{rCRB}, 10 \log _{10} \gamma / R_{D}^{2}=$ $225.81 \mathrm{~dB} \mathrm{~s}^{-1}, K=4, x_{\max }=y_{\max }=10 \mathrm{~m}$, and $\zeta=0.5$.

real-time positioning is possible.

Finally, we compare the rCRB with the root of the MSE (rMSE) of the ML estimator for $\left(x_{U}, y_{U}\right)$. The ML estimator reduces to

$$
\left(\hat{x}_{U}, \hat{y}_{U}\right)=\arg \min _{\left(x_{U}, y_{U}\right)}\left\|\mathbf{r}-R_{p} \tilde{\mathbf{H}} \boldsymbol{\mu}\right\|^{2} .
$$

The cost function to be minimized is a complex function of the receiver position, so that no closed-form expression for the ML estimate can be obtained. However, it can be verified that the cost function is well behaved and has a single minimum. To simulate the performance of the ML estimator, we use the Optimization Toolbox from Matlab to find $\left(\hat{x}_{U, M L}, \hat{y}_{U, M L}\right)$, and compute the root of the MSE on the position, $r M S E_{M L}=\left(E\left[\left(x_{U}-\hat{x}_{U, M L}\right)^{2}+\left(y_{U}-\right.\right.\right.$ $\left.\left.\left.\hat{y}_{U, M L}\right)^{2}\right]\right)^{1 / 2}$. The rMSE, averaged over all positions in a $5 \mathrm{~m} \times 5 \mathrm{~m}$ room, is shown in Fig. 10 as function of the ratio $\gamma$ (23), which is proportional to the signal-to-noise ratio, together with the corresponding rCRB. We consider two situations. In the first situation, only a LOS component is considered, i.e. no reflections are present, whereas in the second situation, also a 
diffuse channel component is present, due to reflections of the signals on the walls. The diffuse component will introduce an additional term $R_{p} \tilde{\mathbf{H}}_{N L O S} \boldsymbol{\mu}$ in (2), i.e.

$$
\mathbf{r}=R_{p} \tilde{\mathbf{H}} \boldsymbol{\mu}+R_{p} \tilde{\mathbf{H}}_{N L O S} \boldsymbol{\mu}+\tilde{\mathbf{n}}
$$

where $\left(\tilde{\mathbf{H}}_{N L O S}\right)_{j, i}=h_{c, N L O S}^{(j, i)}$. We simulated the diffuse channel gain $h_{c, N L O S}^{(j, i)}$ by dividing the reflecting surfaces into small areas and calculating the light received by each small area from a given LED. The area was then considered as a Lambertian transmitter [18] with total transmit power proportional to the received power and the reflectivity of the surface. The power received by a given RE from this Lambertian transmitter was calculated using (4)-(6). The total diffuse component was calculated by summing the contributions from all reflecting surfaces. We took into account the first order reflections only. Following [26], a reflection coefficient $\rho=0.5$ was used for the walls. When only a LOS component is present, the performance of the ML estimator is close to the CRB, which indicates the CRB is a valuable tool for evaluating the performance if only a LOS component is present. For the case where a diffuse component is present, we use the ML estimator (24), which does not take into account the presence of a diffuse component $\tilde{\mathbf{H}}_{N L O S}$ in the computation of $R_{p} \tilde{\mathbf{H}} \mu^{4}$. As can be observed, the performance of the estimator shows an error floor for large $\gamma$. The error floor for case 1 is larger than for case 2. To further investigate this error floor, we show in Fig. 11 the rMSE of the ML estimator with and without diffuse component, as function of $y_{D}$, for $x_{D}=0$, where $\left(x_{D}, y_{D}\right)=(0,0)$ is the center of the room. As can be observed in the figure, the rMSE curves with and without diffuse component are very close in the middle of the room, as long as the position of the receiver is within the square with as corner points the LEDs (i.e. $y_{D}<1.25 \mathrm{~m}$ ). This can be explained as follows. Within this square, the LOS components of all REs and LEDs are sufficiently large, whereas the diffuse component is small as the receiver is far from the walls. The closer the receiver is to a wall, the larger the diffuse component due to that wall will be, while the LOS component to the LEDs that are furthest away reduces, due to the directivity of the REs. Hence, for these LEDs, the diffuse component will become dominant for some of the REs. In case 1, the RSS values corresponding to the different LEDs are correlated, i.e. the matrix $\mathbf{R}_{s}$ is not diagonal. Hence, the adverse effect of the dominant diffuse component for the LEDs that are further away from the receiver will also affect the RSS values from the nearby LEDs. Because in case 2, the contributions from the different LEDs are uncorrelated (i.e. $\mathbf{R}_{s}$ is diagonal), the adverse effect of the dominant diffuse

\footnotetext{
${ }^{4}$ The diffuse component depends on the positions of the LEDs and the receiver w.r.t. the walls, as well as on the angle-of-arrival of a reflection arriving at the receiver. The ML estimator that takes into account the presence of the position-dependent diffuse component requires the prior knowledge of the dependency of the diffuse component to the position. However, as no simple but accurate analytical model for this diffuse component as function of the position and the angle-of-arrival exists, the ML estimator that is optimal for the case with diffuse component, i.e. that uses the knowledge of the dependency of the diffuse component $\tilde{\mathbf{H}}_{N L O S}$ on. the position of the receiver and the angle-of-arrival of a reflection in the computation of $R_{p} \tilde{\mathbf{H}} \boldsymbol{\mu}$ in (24), can not be derived.
}

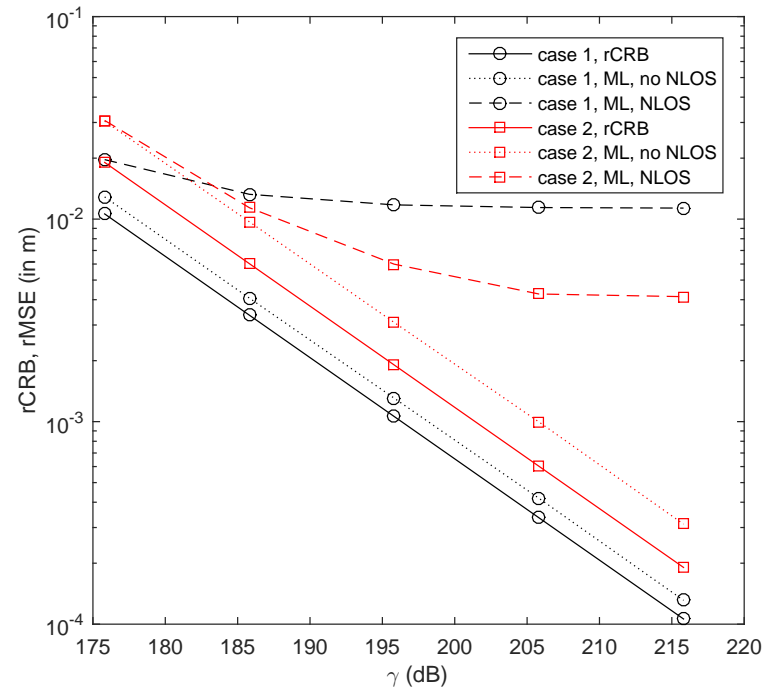

Fig. 10. Comparison of $\operatorname{rMSE}_{M L}$ and $\mathrm{rCRB}, K=4, x_{\max }=y_{\max }=5 \mathrm{~m}$, $h_{A}=R_{D}, \zeta=0.5$ and $\rho=0.5$.

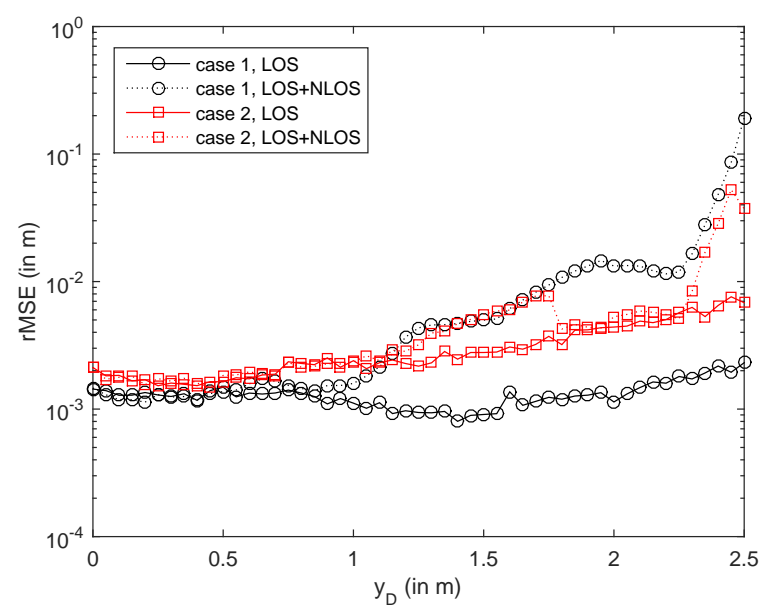

Fig. 11. $\operatorname{rMSE}_{M L}$ with and without diffuse component as function of the position $y_{D}$ (with $x_{D}=0$ ), $\gamma=195.81 \mathrm{~dB}, K=4, x_{\max }=y_{\max }=5 \mathrm{~m}$, $h_{A}=R_{D}, \zeta=0.5$ and $\rho=0.5$.

component will not affect the contributions from the nearby LEDs, so the performance degradation for case 2 is smaller. For case 2, only when the receiver is very close to the wall, the performance degradation is substantial, although the resulting positioning error is smaller than $10 \mathrm{~cm}$. Hence, even in the presence of diffuse reflection of the light, centimetre accuracy can be obtained. Our results show that, for most positions in the room, the gap between the MSE of the ML estimator and the CRB is small. This indicates that the CRB, which was derived for the case of a LOS component only, is still a suitable lower bound on the performance even if diffusion, due to reflections on the walls and objects, is present. As the computational complexity of the $\mathrm{CRB}$ is much lower than simulating the ML estimator performance, the CRB is well suited to evaluate the efficiency of practical estimators and optimize the system parameters. 


\section{CONClusions \& Discussion}

In this paper, we investigate the Cramer-Rao bound for positioning using visible light. In contrast to other works dealing with the Cramer-Rao bound for visible light positioning, we consider a directional receiver [13] combined with nondirectional LEDs. In our approach, we correlate the outputs of the PDs with a set of reference signals related to the signals transmitted by the LEDs, in order to separate the contributions from the different LEDs. Based on the resulting observations, the $\mathrm{CRB}$ on the position error is determined. This CRB depends on the transmitted signals and the reference signals. Therefore, we consider two different cases, where in the first scenario, the received signal is correlated with the transmitted optical signals, and in the second scenario, the dc-component is removed from the received signals. The CRB is compared with the MSE of the ML estimator, and it is shown that the MSE closely matches the CRB. The CRB, which was derived for the case of a LOS component only, is still a good lower bound for the case where diffuse reflections, due to reflections of the light on walls or objects, are present, except for positions near these walls. Further, the CRB can be computed with low complexity, in contrast to the evaluation of the performance of the ML estimator. This illustrates the relevance of the CRB. Further, we compare the results with the CRB derived in [17]. The first scenario gives the better performance, as all available information is used, and outperforms [17], where only the dccomponent is kept. Our numerical results show that accurate real-time positioning is possible with a compact directional receiver, using only a limited number of LEDs per room. As a rule of thumb, we found that one LED per $25 \mathrm{~m}^{2}$, used for positioning, is sufficient to obtain centimetre accuracy.

In practice, the excellent performance obtained in this paper will be degraded by system imperfections, such as the differences in dc-offsets at the transmitter and receiver, and imperfections in the sizes, shapes and positions of the PDs and the apertures. In this paper, we already considered a solution to deal with the unknown dc-offset at the transmitter. By correlating the received signals with the carrier component of the transmitted signal (i.e., Case 2), the unknown dccomponent is removed from the signal, at the cost of a slight reduction of the position accuracy. With respect to the other system impairments, we expect that the resulting accuracy will be of the same order of magnitude as UWB suffering from multipath interference (i.e. better than $10 \mathrm{~cm}$ ), which is an order of magnitude better than the position accuracy that can be obtained with WiFi or BLE solutions (i.e. 2-3 m).

\section{APPENDIX}

In this appendix, we derive the FIM of the receiver position $\boldsymbol{\theta}=\left(x_{U}, y_{U}\right)$, based on the observation $\mathbf{r}$ (2). Taking into account that $\mathbf{r} \mid \boldsymbol{\theta} \sim N\left(R_{p} \tilde{\mathbf{H}} \boldsymbol{\mu}, \frac{N_{0}}{2} \tilde{\mathbf{R}}\right), \ln p(\mathbf{r} \mid \boldsymbol{\theta})$ can be written as

$$
\ln p(\mathbf{r} \mid \boldsymbol{\theta})=C-\frac{1}{N_{0}}\left(\mathbf{r}-R_{p} \tilde{\mathbf{H}} \boldsymbol{\mu}\right)^{T} \tilde{\mathbf{R}}^{-1}\left(\mathbf{r}-R_{p} \tilde{\mathbf{H}} \boldsymbol{\mu}\right),
$$

where $C$ is an irrelevant constant. Hence, the element $(a, b)$ of the FIM, i.e. $\left(\mathbf{F}_{U}\right)_{a b}$ can be written as

$$
\begin{aligned}
\left(\mathbf{F}_{U}\right)_{a b} & =E_{\mathbf{r}}\left[\left(\frac{\partial}{\partial a} \ln p(\mathbf{r} \mid \boldsymbol{\theta})\right)\left(\frac{\partial}{\partial b} \ln p(\mathbf{r} \mid \boldsymbol{\theta})\right)^{T}\right] \\
& =\frac{2 R_{p}^{2}}{N_{0}} \boldsymbol{\mu}^{T}\left(\frac{\partial}{\partial a} \tilde{\mathbf{H}}\right)^{T} \tilde{\mathbf{R}}^{-1}\left(\frac{\partial}{\partial b} \tilde{\mathbf{H}}\right) \boldsymbol{\mu} .
\end{aligned}
$$

Taking into account that

$$
\frac{\partial}{\partial a} \tilde{\mathbf{H}}=\left(\begin{array}{cccc}
\frac{\partial}{\partial a} \mathbf{H} & \mathbf{0} & \ldots & \mathbf{0} \\
\mathbf{0} & \frac{\partial}{\partial a} \mathbf{H} & \ldots & \mathbf{0} \\
\vdots & \vdots & & \vdots \\
\mathbf{0} & \mathbf{0} & \ldots & \frac{\partial}{\partial a} \mathbf{H}
\end{array}\right)
$$

and

$$
\tilde{\mathbf{R}}^{-1}=\left(\begin{array}{ccc}
\left(\mathbf{R}_{\psi}^{-1}\right)_{1,1} \mathbf{I}_{M} & \ldots & \left(\mathbf{R}_{\psi}^{-1}\right)_{1, L} \mathbf{I}_{M} \\
\vdots & & \vdots \\
\left(\mathbf{R}_{\psi}^{-1}\right)_{L, 1} \mathbf{I}_{M} & \ldots & \left(\mathbf{R}_{\psi}^{-1}\right)_{L, L} \mathbf{I}_{M}
\end{array}\right)
$$

it follows that $\left(\mathbf{F}_{U}\right)_{a b}(27)$ can be rewritten as

$$
\left(\mathbf{F}_{U}\right)_{a b}=\frac{2 R_{p}^{2}}{N_{0}} \sum_{\ell, \ell^{\prime}=1}^{K}\left(\mathbf{R}_{\psi}^{-1}\right)_{\ell, \ell^{\prime}} \boldsymbol{\mu}_{\ell}^{T} \mathbf{X}^{a b} \boldsymbol{\mu}_{\ell^{\prime}}
$$

where $\mathbf{X}^{a b}$ is defined in (11). Defining $\left(\mathbf{W}^{a b}\right)_{\ell, \ell^{\prime}}=$ $\boldsymbol{\mu}_{\ell}^{T} \mathbf{X}^{a b} \boldsymbol{\mu}_{\ell^{\prime}}$, and taking into account that $\operatorname{trace}\left(\mathbf{R}_{\psi}^{-1} \mathbf{W}^{a b}\right)=$ $\sum_{\ell, \ell^{\prime}=1}^{L}\left(\mathbf{R}_{\psi}^{-1}\right)_{\ell, \ell^{\prime}}\left(\mathbf{W}^{a b}\right)_{\ell, \ell^{\prime}}$, we obtain (9).

\section{REFERENCES}

[1] T. Komine, M. Nakagawa, "Performance Evaluation of Visible-Light Wireless Communication System Using White LED Lightings," in Proc. 9th Symposium on Computers and Communications (ISCC2004), Alexandria, Egypt, Jun 2004, pp. 258-263.

[2] J. Armstrong, Y. A. Sekercioglu and A. Neild, "Visible Light Positioning: A Roadmap for International Standardisation,' IEEE Communications Magazine, Vol 51, No 12, Dec 2013, pp. 68-73.

[3] S. Hann, J.-H. Kim, S.-Y. Jung, C.-S. Park, "White LED Ceiling Lights Positioning Systems for Optical Wireless Indoor Applications," in Proc. ECOC 2010, Torino, Italy, Sep 2010.

[4] S.-Y. Jung, S. Hann, C.-S. Park, "TDOA-Based Optical Wireless Indoor Localization Using LED Ceiling Lamps," IEEE Transactions on Consumer Electronics, Vol 57, No 4, Nov 2011, pp. 1592-1597.

[5] K. Panta, J. Armstrong, "Indoor Localization Using White LEDs," Electronics Letters, Vol 48, No 4, Feb 2012, pp. 228-230.

[6] A. Arafa, X. Jin, R. Klukas, "Wireless Indoor Optical Positioning With a Differential Photosensor," IEEE Photonics Technology Letters, Vol 32, No 14, Jul 2012, pp. 2480-2485.

[7] S.-Y. Jung, S. Hann, S. Park, C.-S. Park, "Optical Wireless Indoor Positioning System Using Light Emitting Diode Ceiling Lights," Microwave and Optical Technology Letters, Vol 54, No 7, Jul 2012, pp. 1622-1626.

[8] M. Rahaim, G.B. Prince, T.D.C. Little, "State Estimations and Motion Tracking for Spatially Diverse VLC Networks," in Proc. Globecom Workshops, Anaheim, CA, USA, Dec 2012, pp. 1249-1253.

[9] H.-S. Kim, D.-R. Kim, S.-H. Yang, Y.-H. Son, S.-K. Han, "An Indoor Visible Light Communication Positioning System Using a RF Allocation Technique," Journal of Lightwave Technology, Vol 31, No 1, Jan 2013, pp. 134-144.

[10] U. Nadeem, N.U. Hassan, M.A. Pasha, C. Yuen, "Highly Accurate 3D Wireless Indoor Positioning System Using White LED Lights," Electronics Letters, Vol 50, No 11, May 2014, pp. 828-830.

[11] G. Kail, P. Maechler, N. Preyss, A. Burg, "Robust Asynchronous Indoor Localization Using LED Lighting," IEEE International Conference on Acoustics, Speech and Signal Processing (ICASSP2014), Florence, Italy, May 2014. 
[12] S.-H. Yang, H.-S. Kim, Y.-H. Son, and S.-K. Han, "Three-Dimensional Visible Light Indoor Localization Using AOA and RSS With Multiple Optical Receivers," Journal of Lightwave Technology, Vol 32, No 14, Jul 2014, pp. 2480-2485.

[13] T.Q. Wang, C. He, J. Armstrong, "Angular Diversity for Indoor MIMO Optical Wireless Communications," in Proc. IEEE International Conference on Communications (ICC2015), London, UK, Jun 2015.

[14] X. Zhang, J. Duan, Y. Fu, A. Shi, "Theoretical Accuracy Analysis of Indoor Visible Light Communication Positioning System Based on Received Signal Strength Indicator," IEEE Journal of Lightwave Technology, Vol 32, No 21, Nov 2014, pp. 3578-3584.

[15] A. Sahin, Y.S. Eroglu, I. Guvenc, N. Pala, M. Yuksel, "Hybrid 3D Localization for Visible Light Communication Systems," Journal of Lightwave Technology, Vol 33, No 22, Nov 2015, pp. 4589-4599.

[16] T.Q. Wang, Y.A. Sekercioglu, A. Neild, J. Armstrong, "Position Accuracy of Time-of-Arrival Based Ranging Using Visible Light with Application in Indoor Localization Systems," Journal of Lightwave Technology, Vol 31, No 20, Oct 2013, pp. 3302-3308.

[17] H. Steendam, T.Q. Wang, J. Armstrong, "Cramer-Rao Bound for Indoor Visible Light Positioning Using an Aperture-Based Angular Diversity Receiver," in Proc. ICC'2016, Kuala Lumpur, Malaysia, Jun 2016.

[18] J.M. Kahn, J.R. Barry, "Wireless Infrared Communications," Proceedings of the IEEE, Vol 85, No 2, Feb 1997, pp. 265-298.

[19] H.L. Van Trees, "Detection, Estimation, and Modulation Theory," New York, NY, USA, Wiley, 1968.

[20] J.B. Carruthers, , J.M. Kahn, "Multiple-subcarrier modulation for nondirected wireless infrared communication," IEEE Journal on Selected Areas in Communications, Vol 14, No 3, Mar 1996, pp. 538-546.

[21] O. Gonzalez, R. Perez-Jimenez, S. Rodriguez et Al., "OFDM over indoor wireless optical channel," IEE Proceedings - Optoelectronics, Vol 152, No 4, Aug 2005, pp. 199-204.

[22] N.U. Hassan, A. Naeem, M.A. Pasha, C. Yuen, "Indoor positioning using visible LED lights: a survey," ACM Computing Surveys, Vol 48, No 2, Nov 2015, pp. 20.1-20.32.

[23] M. Aminikashani, W. Gu, M. Kavehrad, "Indoor location estimation with optical-based orthogonal frequency division multiplexing communications," Optical Engineering, Vol 55, No 5, May 2016, doi: 10.1117/1.OE.55.5.056116.

[24] E.-M. Jeong, S.-H. Yang, H.-S. Kim, S.-K. Han, ”Tilted receiver angle error compensated indoor positioning system based on visible light communication," Electronics Letters, Vol 49, No 14, Jul 2013, pp. 890891.

[25] L. Zeng, D.C. O'Brien, H.L. Minh, G.E. Faulkner, K. Lee, D. Jung, Y. Oh, E.T. Wong, "High Data Rate Multiple Input Multiple Output (MIMO) Optical Communications Using White LED Lighting," IEEE Journal on Selected Areas in Communications, Vol 27, No 9, Dec 2009, pp. 1654-1662.

[26] O. Bouchet, Wireless Optical Telecommunications, Wiley-ISTE, 2012. 